\title{
Linx
}

Revue des linguistes de l'université Paris X Nanterre

$78 \mid 2019$

La linguistique des genres, en actes et en questions

\section{Traces linguistiques d'interactions avec les genres dans le sonnet romantique "When I have fears » de John Keats}

Linguistic traces of genre dialogue in the romantic sonnet "When I have fears"

by John Keats

\section{Stéphane Kostantzer}

Édition électronique
URL : https://journals.openedition.org/linx/3040

DOI : 10.4000/linx.3040

ISSN : 2118-9692

Éditeur

Presses universitaires de Paris Nanterre

\section{Référence électronique}

Stéphane Kostantzer, «Traces linguistiques d'interactions avec les genres dans le sonnet romantique "When I have fears » de John Keats », Linx [En ligne], 78 | 2019, document 4, mis en ligne le 30 juin 2019, consulté le 29 juillet 2022. URL : http://journals.openedition.org/linx/3040 ; DOI : https://doi.org/ $10.4000 / \operatorname{linx} 3040$

Ce document a été généré automatiquement le 29 juillet 2022

Tous droits réservés 


\title{
Traces linguistiques d'interactions avec les genres dans le sonnet romantique "When I have fears » de John Keats
}

\author{
Linguistic traces of genre dialogue in the romantic sonnet "When I have fears" \\ by John Keats
}

Stéphane Kostantzer

\section{Introduction}

1 Le poème "When I have fears ", écrit en 1818, s'inscrit à la croisée de trois traditions discursives que sont le sonnet, le poème lyrique, le romantisme. Ceci pose au moins deux questions : ces traditions constituent-elles des genres, et si tel est le cas, quel rapport ce texte entretient-il avec ces genres ${ }^{1}$ ?

2 Notre étude forme l'hypothèse que ce poème construit sa signification autour d'un dialogue avec les attendus du sonnet, du lyrisme et du romantisme, et que ce dialogue, qu'il procède par reflet, désaccord ou filiation, présuppose leur conception par l'auteur en tant que genres. Genre sera employé au sens bakhtinien du terme, c'est-à-dire en tant qu'il renvoie à une conceptualisation relativement stabilisée et conscientisée de normes morphosyntaxiques, thématiques et compositionnelles, dérivées d'une lignée d'énoncés. Nous emploierons les outils de la linguistique énonciative pour analyser les observables du texte qui s'apparentent à des traces d'interaction avec ces trois traditions entendues comme genres. L'analyse se concentrera sur les aspects syntaxiques du texte puis sur l'expression des quantités et le traitement particulier dont elles font l'objet, dans le but de dégager ce qui constitue des indices d'une triple filiation générique et, le cas échéant, des écarts propices au développement d'un style personnel. 


\section{Réflexions sur la notion de genre}

3 La notion de genre est indissociable de celle de modèle et de normes. Un genre sélectionne de façon plus ou moins prescriptive des unités significatives, thématiques et morphosyntaxiques, leur organisation selon une certaine dynamique, et incite au réemploi de celles-ci. La reconnaissance d'un genre s'établit à partir de l'attente du respect de ces normes, elles-mêmes conceptualisées à partir d'une lignée d'énoncés. On ne peut concevoir un genre s'il ne possède pas une historicité qui le distingue d'autres genres. Vus sous un angle diachronique, les genres se conçoivent ainsi comme des lignées de réécritures à partir de textes qui font événement (Rastier \& Pincemin, 1999), et ne cessent de se recréer, par imbrication, interpolation, conflit, absorption, ou coalescence (Koch, 1997). Ces relations complexes font qu'une fois un certain degré de stabilité et d'abstraction atteint, un genre devient catégorisable, selon des facteurs d'historicité : romantisme allemand, britannique (voir Duff, 2009), ou morphosyntaxiques: sonnet pétrarquiste, spencerien, shakespearien (voir Spiller, 1992). Le degré de conscientisation, voire de théorisation, qui peut entourer les normes et l'histoire d'un genre apparaît alors comme un élément définitoire majeur de ce qui fait genre.

4 Cette première définition du genre pose néanmoins quelques problèmes. Le poème à l'étude est un sonnet romantique lyrique. S'agit-il d'un genre en soi, ou sommes-nous face à un texte affilié simultanément à trois genres? En effet, comme nous le verrons en deuxième partie, chacune de ces trois entrées renvoie à des corrélations saillantes et discriminantes décelables par l'analyse morphosyntaxique, et chacune s'inscrit dans une lignée historique. Cette dernière donnée est en grande partie responsable du fait que certains thèmes leur sont associés. Un autre problème concerne le statut des thèmes récurrents. La seule récurrence d'un thème ne préjuge pas de son éventuel statut générique. Ainsi, on peut douter de ce statut si le thème s'avère exploité de façon transversale dans d'autres genres contemporains. Il serait alors plutôt de nature idéologique que générique ${ }^{2}$. Le concept de topos (Anscombre, 1995: 86), entendu comme « croyance » servant de « support du discours argumentatif » permet de mettre à jour non plus seulement des thèmes, mais des thématiques, que nous entendrons comme unités constituées d'un thème auquel est associé une argumentation (une dialectique ou une problématique). Le fait que la récurrence concerne une thématique plutôt qu'un thème pourrait favoriser son rattachement à un genre plus qu'à une doxa, bien que cela reste matière à débats. La conception bakhtinienne du genre permet-elle de résoudre ces premiers problèmes?

5 Les théories de Bakhtine invitent à s'intéresser à la façon dont les réécritures successives entrent en dialogue entre elles et/ou avec le modèle fondateur d'un genre. Tout comme la langue s'appréhende par la réalité des discours, la conceptualisation d'un genre se construit à partir de la mise en rapport de textes. Les interactions des textes littéraires ne sont pas différentes des interactions verbales du quotidien, qui s'appuient tout autant sur l'utilisation de genres. Bakhtine emploie le terme de genres discursifs pour désigner les sphères d'échange qui structurent l'interaction verbale, de la même façon que grammaire et langue organisent la parole (Bakhtine, 1984). Dans cette perspective, tout énoncé s'inscrit dans un genre (conversationnel, argumentatif, etc.), et le genre s'invite au cœur de la signification, laquelle selon Anscombre (1995: 
33) renvoie moins aux objets du monde extérieur qu'à des discours dont l'énoncé est la continuation. En somme, le sens se construit par rapport à d'autres sens, et toujours d'après un genre.

6 Le concept bakhtinien de dialogisme ne concerne ainsi pas seulement les rapports interpersonnels, mais aussi les rapports entre un texte et le ou les genres dans le(s)quel(s) il s'inscrit. Un texte est avant tout un énoncé, qui selon Bakhtine, est une réplique. Il répond à d'autres énoncés de façon polémique, parodique, ou par stylisation (imitation, adhésion ou transformation) : « un énoncé établit des liens dialogiques - de reflet, de désaccord, de filiation - avec les autres énoncés de la sphère de la communication discursive à laquelle il appartient »(Grillo, $2007: 22)$. Le dialogisme bakhtinien concerne les subjectivités, mais aussi les idéologies, et les genres.

7 Les genres suggèrent ou imposent des normes compositionnelles (longueur, ordre, structure, mode, etc.), stylistiques (favorisation de moyens lexicaux ou grammaticaux), contraints en partie par ces premiers, ainsi que des contenus thématiques (par exemple, la saillance de l'introspection propre au lyrisme). Ces normes permettent de formaliser l'anticipation dialogique des attentes du destinataire, et conditionnent l'expressivité et le style personnel de l'auteur.

8 La théorie de Bakhtine, qui grâce à un continuum entre imitation et écart permet de mettre à jour des interactions génériques à la plus petite échelle de l'énoncé, s'est développée en réaction aux analyses immanentes préconisées par les partisans d'une poétique formaliste qui tenaient le texte pour autosuffisant (Morris, 1994 : 124). Pour Bakhtine, le dialogue générique permet d'analyser le rôle des genres dans la production de l'énoncé, et pas seulement de se servir des genres pour simplement classer une œuvre dans une tradition. Mais si on conçoit aisément les avantages à étendre la portée du concept de genre (par exemple, il peut dans cette vue ne concerner qu'une phrase d'un énoncé, voire un mot), on conçoit aussi la réticence à nommer genres des genres dont l'histoire et les normes paraissent extrêmement vastes. Pour beaucoup, la fiction, notamment parce qu'elle trouve un corolaire en langue (le plan du contrefactuel), s'apparente davantage à un mode qu'à un genre. La poésie est plus souvent qualifiée d'art, peut-être du fait que son seul principe fédérateur semble être la fonction esthétique: superposition des sens dans une "économie permanente de moyens d'expression " (Fonagy, 1966: 110). Le sonnet est parfois vu comme une forme compositionnelle ("a prescribed form», Spiller, 1992:2). Malgré une historicité très forte (les sonnets se répondent) et une esthétique commune, cette étiquette est choisie par Spiller du fait que le sonnet brasse des thématiques trop disparates. Quant au romantisme, il est assez souvent décrit comme une esthétique, un courant ou une idéologie. Enfin, le lyrisme figure assez communément en tant que mode d'expression (la subjectivité de la première personne), en opposition au mode épique.

Qu'elles soient conçues en tant que genres ou non, ces entrées constituent des systèmes plus ou moins normatifs rassemblés a minima par une unité fonctionnelle ou esthétique (un même but, un même instrument), déduits à partir d'une collection d'occurrences individuelles historiques.

\section{Sonnet, lyrisme, romantisme en tant que genres}

10 Le sonnet est une forme contraignante. Spiller (1992:3) reste prudent en ce qu'il accepte de considérer sonnet tout poème qui respecterait au moins l'un de ces trois 
principes : «it has proportion, being in eight and six, and extension, being in ten- or eleven-syllable lines, and duration, having fourteen of them. " Le poème de Keats reprend le schéma $A B A B C D C D$ EFEF GG inventé par Surrey au XVIème siècle : trois quatrains et un couplet, dix pieds par vers. Cette forme impose concision et compacité. Elle ne favorise pas la narration, mais plutôt l'exposition structurée d'une pensée ou d'une expérience unique qui évolue puis trouve une conclusion à l'occasion des deux derniers vers, souvent le lieu d'une recherche finale de clôture par le consensus, la sagesse populaire ou le mot d'esprit.

11 Expliquées dans Spiller (1992 : 125), ses affinités avec le lyrisme, constatées depuis les sonnets de Pétrarque, passent par un mode d'expression personnelle à portée universelle, une dialectique entre une voix qui expose le sentiment et une autre qui le déplace sur un plan explicatif, voire philosophique - un moi introspectif, qui se confie et s'exprime souvent dans la souffrance, et un moi argumentatif, plus détaché. Le topos typique du lyrisme est la difficulté d'accéder à sa propre conscience, un topos qui se superpose parfaitement à l'un des topoï du sonnet, notamment le sonnet pétrarquéen (Spiller 1992: 18, 72). La forme du sonnet favorise la composition dialectique. La récurrence de certains topoï légitime de considérer le sonnet comme un genre plutôt qu'une forme, même si sa longue histoire rend difficile la distinction entre topoï génériques et idéologiques. Les topoï suivants sont reconnus chez Spiller (1992) ou Duff (2009) comme récurrents et se retrouvent dans notre texte : l'amour est empêché ou non partagé (Pétrarque, premiers sonnets italiens). L'être aimé est à la fois sensualisé et idéalisé. Non soumis à la mutabilité, dépourvu d'attributs physiques précis, l'être aimé symbolise la grâce ou le divin (le sonnet des stilnovisti). Dépeint comme un adversaire ou un idéal de perfection au contact duquel le moi se transforme, il ne sert que comme surface réfléchissante des états d'esprit évolutifs d'un amoureux épris et contemplatif. De façon similaire, la beauté de la nature, lorsque dépeinte, symbolise l'impact de l'amour sur soi. Un autre topos prend pour thème l'acte d'écriture, qui fait souvent l'objet d'allusions métatextuelles, et dont la problématisation peut aboutir aux thématiques suivantes: le sujet se représente en tant qu'il lance un appel à l'inspiration ; il fait aveu de faiblesse quant à la réussite de l'expression de sa pensée ; il confesse ses difficultés à résoudre le paradoxe de l'ineffable (comment réussir, par le langage, à exprimer l'échec du langage). Le moi est universel. Il est tourmenté par la dynamique du désir, qui provient d'un sentiment d'absence, absence que l'acte d'écriture vise à abolir. Le temps impose mutabilité, il est source à la fois de terreur (mortalité) et d'espoir : le poète recherche la célébrité (fonction utilitaire originelle des premiers sonnets italiens par les courtisans), il cherche à persuader son lecteur.

12 Le romantisme, s'il est un genre, posséderait lui aussi ses thématiques propres. Mouvement historiquement conçu en réaction à la surpuissance des sciences et de la raison telle qu'elle fut consacrée par la Révolution Industrielle (Lakoff, 2003 : 191), le romantisme anglais apparaît à l'origine comme un courant visant à promouvoir l'imagination. L'idéal visé étant de succomber passivement à la beauté de la nature en cherchant à se fondre en elle (Lakoff, 2003 : 229). Le romantisme se définit par une hyperconscientisation de la question des genres, comme l'ont théorisé les romantiques allemands. Aussi de nombreux poèmes romantiques font-ils allusion à l'acte d'écriture. Auto-proclamée forme d'art ultime, le romantisme se voulait au moins un genre totalisant, ce qui le rend délicat à définir en tant que tel. Il théorise une esthétique d'incorporation d'anciens genres, conçus comme modes de pensée plutôt que comme 
principes formels, qu'il s'agissait de rajeunir, d'unir de façon organique et fertile plutôt que de mettre en conflit, dans le but de transcender la question du genre (Duff, 2009).

Plutôt que d'énumérer l'ensemble extrêmement vaste des topoï du romantisme en tant que genre, nous proposons de lister ici ceux qui d'une part se retrouvent dans le texte à l'étude, et qui, surtout, s'accommodent parfaitement de certaines normes du genre du sonnet. La rigidité formelle du sonnet offre au poète romantique un cadre idéal pour exprimer la tension inhérente aux topoï romantiques afférant au chaos de l'émotion, tout en lui imposant de clore sur un instant d'équilibre ou de lucidité. Ainsi, par son caractère épiphanique, le topos du sublime (posé comme une admiration si forte qu'elle dépersonnalise le sujet) s'accommode parfaitement de la contrainte de condensation du sonnet. Les topoï génériques du romantisme qu'on retrouve dans notre texte sont selon nous classables en deux rubriques. Une première série affaire à un topos général qui fait du même un différent : la passion et l'imagination prévalent sur la raison. L'énergie du désir se confronte à une tentative de contrôle (au moins par la forme). Les éléments de la nature (terre, eau, feu, air, végétal) se détachent tout en étant dépeints dans leur harmonie, tandis que se problématisent les interactions entre les hommes, la nature, et parfois le divin. Le temps linéaire et orienté de la mortalité s'oppose au temps cyclique ; les points de vue sur un même objet se multiplient (l'intérieur devient un extérieur, le temps devient espace, l'agent devient patient). Le topos général inverse, qui fait que le différent devient un même, se voit illustré par les rapprochements entre l'éphémère et l'éternel ; le micro- et le macroscopique ; le 'je' et le 'vous'. S'inscrivent également dans ce paradigme la stratégie du haut degré, par laquelle le sublime, l'abondance et l'exacerbation peuvent frapper uniformément tout objet décrit, y compris l'ordinaire et le modéré ; les réversibilités axiologiques, qui font que la souffrance devient une joie, que l'absence suscite la création; et enfin, une vue qui tente d'opérer une réconciliation entre nostalgie, voire archaïsme, et innovation.

Face à ces vastes problématiques, l'ambition de cet article sera tout d'abord de tenter de définir les principes compositionnels de ce texte et ses spécificités syntaxiques qui permettraient de l'affilier à des genres; partir des observables du texte en tant qu'énoncé pour isoler de possibles éléments révélateurs d'une conscience générique ; tenter de rapprocher les thématiques du texte à des topoï caractéristiques de genres existants, et dans le même temps, tenter de mettre à jour ceux qui l'en distinguent. L'hypothèse est que l'énoncé conserve les accents d'un dialogue avec un amont textuel, lequel se laissera ou non appréhender comme catégories génériques, selon trois traditions imbriquées que sont le romantisme, le lyrisme, le sonnet. Afin de mettre à jour de tels accents, nous proposons de nous pencher sur les caractéristiques syntaxiques du texte, lesquelles nous amèneront dans un second temps à nous intéresser au traitement linguistique général de la quantité.

\section{Analyse syntaxique}

When I have fears that I may cease to be

Before my pen has glean'd my teeming brain,

Before high-piled books, in charact'ry,

Hold like rich garners the full-ripen'd grain;

When I behold, upon the night's starr'd face,

Huge cloudy symbols of a high romance,

And think that I may never live to trace

Their shadows, with the magic hand of chance; 
And when I feel, fair creature of an hour!

That I shall never look upon thee more,

Never have relish in the faery power

of unreflecting love!-then on the shore

of the wide world I stand alone, and think

Till Love and fame to nothingness do $\operatorname{sink}^{3}$. starr'd face) est un attribut locatif qui localise huge cloudy symbols. Il peut être supprimé ou déplacé sans altération de sens. Y figure un génitif déterminatif qui marque un repérage de type possession, présenté de façon intégrée par le choix de la structure N's $N$ plutôt que $N$ of $N$. Of figure justement dans le second SP, of a high romance, qui complète le nom symbols. Of encode sur un mode synthétique une relation prédicative sous-jacente qui n'est pas ici de l'ordre de la possession, mais plutôt de l'attribution : symbols identifiable to a high romance. Une seconde relation prédicative est ajoutée par coordination, toujours sous la portée de la temporelle. And think [...] décrit une réflexion consécutive à la contemplation dite par When I behold [...], qui en découle également de façon logique. And think that I may never live to trace / Their shadows intègre une deuxième proposition nominale en that, complétant cette fois un verbe (think). À l'intérieur figure une subordonnée infinitive (to trace [...]) complément du verbe live. Le complément to trace their shadows est un argument du verbe, il est complément nécessaire du verbe caténatif live. Le sens s'établit à partir de I may never trace their shadows, sur lequel live to, partiellement fossilisé, apporte une contribution aspectuelle ou modale (proche de be able to trace) ; to peut encoder une intentionnalité mais n'introduit pas une circonstancielle de but ${ }^{4}$.

19 Le dernier SP, with the magic hand of chance, en imbrique un autre. À valeur circonstancielle d'instrument, with the magic hand of chance se rattache au verbe trace.

Par le sémantisme de with, la main passe d'agent à instrument (à comparer à before my temps introduites par le subordonnant when. La première occupe les quatre premiers vers, la deuxième les trois suivants, et la dernière débute au vers 9 et se termine au milieu du vers 12. La principale, introduite par l'adverbe then, lui aussi temporel, débute après un tiret et s'étend sur seulement deux vers. Une quatrième circonstancielle de temps, introduite par Till conclut le poème.

La syntaxe de la première circonstancielle est complexe : une proposition nominale en that vient tout d'abord offrir une postmodification au nom fear. À l'intérieur figurent le modal épistémique may et une subordination nominale infinitive to be, complément de cease. Depuis les travaux de Perlmutter (1970), et jusqu'à très récemment (Girard-Gillet 2016), on considère qu'un verbe aspectuel tel que cease, dès lors qu'il ne sélectionne pas ses arguments, est un verbe opérant une montée du sujet. Ici, ce qui peut cesser, ce n'est pas I, mais son existence, autrement dit, c'est la relation prédicative entière $<I$ / be>. L'énoncé sous-jacent est ainsi : <I / be> cease. La montée du sujet I promeut son référent en tant que thème, et le place métriquement au centre du premier vers. Elle le met en saillance, conformément aux attendus du genre lyrique. Suppressible, mais fortement collocationnel avec fears, may modalise ensuite cette relation prédicative: $<$ / be> may cease. 
pen has glean'd my teeming brain). Le SP of chance est à valeur génitive. Le lien d'appartenance, posé par $N$ of $N$ plutôt que $N$ 's $N$, est présenté comme plus lâche, moins préconstruit, ou moins congruent, que celui tissé dans the night's starr'd face. Mais pourquoi le fait que la nuit ait un visage serait-il plus congruent que le fait que le hasard possède une main? Les deux sont des entités inanimées que le locuteur personnifie, mais of maintient l'autonomie référentielle de hand, alors que $N$ 's $N$ déclasse celle de face. Bien que toutes deux soient des parties du corps, hand jouit par contraste d'une saillance, qui facilite l'activation du topos afférant à la difficulté de dépeindre (hand) ses émotions. Face, rendu syntaxiquement moins saillant, active celui qui pose de rendre généraux les attributs de la beauté féminine, de les mêler à ceux de la nature (night's) de sorte qu'ils servent surtout de support réfléchissant les émotions du sujet contemplatif. Ce jeu discret entre les deux types de génitif prendrait alors une nouvelle dimension, chaque structure servant à activer un topos différent, mais propre au sonnet.

La troisième proposition circonstancielle de temps, And when I feel [...] est interrompue par une incise : fair creature of an hour. Ce syntagme nominal apposé est-il co-référent à $I$ ou est-ce une adresse au co-locuteur intra-diégétique ? Si l'on commutait fair avec un qualificatif non mélioratif, l'interprétation co-référente s'imposerait: And when I feel, [poor] creature of an hour [that I am]. Associé à creature, fair ne signifie plus " agréable " ou "juste ", mais s'emploie en poésie pour signifier «beau ", et parfois «blond». De son côté, creature qualifié de fair prend à de nombreuses reprises chez Shakespeare le trait [+féminin] (Bartlett, 2016 : 482). L'assemblage s'emploie dans la tradition poétique pour exprimer un haut degré de beauté féminine, un degré bien supérieur à celui que dénotent les termes dans l'absolu. C'est bien ici le genre poétique qui active l'interprétation méliorative, qui se charge de spécifier un sens parmi le faisceau de significations possibles de chacun des deux termes et donne la clé de lecture de la figure de style (interprétation méliorative derrière l'euphémisme), ce qui au final renseigne sur la valeur locutoire de l'apposition, qui ainsi dépend étroitement de ce paramètre générique.

21 Une proposition nominale en that complète feel. Elle se constitue de deux relations prédicatives coordonnées par une virgule. La seconde, qui fait l'ellipse du sujet $I$ et du modal shall, instancie un nouveau prédicat associé à I : Never have relish in the fairy power / Of unreflecting love. Le modal, ici à valeur épistémique, encode un degré de probabilité accru par rapport à may. Il possède des invariants que sont la non-congruence (Lapaire et Rotgé, 1991), et la non-autonomie du sujet (Bouscaren, 1987). Ces valeurs convergent pour signifier le caractère inéluctable de la validation, que l'adverbe more en association à never projette dans un avenir absolu. Une reformulation serait : that I will never look upon thee more is ineluctable. La valeur d'inéluctabilité, qui peut parfois revêtir un caractère prophétique, pourrait renvoyer à un topos lyrique : le sujet est dépeint comme désagentivé, soumis au temps, tout en restant saillant grâce à la thématisation que permet l'emploi de shall en rivalité avec une modalité externe du type it is ineluctable that .... On peut aussi lire dans la progression de may cease à shall never look la trame d'une évolution qui se joue dans la subjectivité du protagoniste, ses certitudes, sa perception du temps. Cette progression de la conscience, et non des événements, typique du sonnet et du lyrique, se lit également dans le sémantisme des deux termes look et relish, qui vont de [+objectif] à [+subjectif]. 

intègre le complément du verbe prépositionnel. Les deux autres s'emboîtent dans : have relish in the fairy power of unreflecting love. L'unité lexicale have relish in constitue un verbe prépositionnel. Le second SP, dans la structure $N$ of $N$ the faery power of unreflecting love, synthétise une relation sous-jacente qui n'exprime pas une simple possession : the faery power that unreflecting love exerts. La manipulation par N's $N$ est de ce fait peu heureuse: ?unreflecting love's faery power. L'usage consacre le génitif roman dans the power of love, car bien que préconstruit, le lien s'établit sur la base d'une propriété prédicative (love has a power/grip on people). of maintient l'autonomie référentielle des termes qu'il unit, ce qui facilite l'activation de topoï quasi proverbiaux : « le pouvoir de l'amour est infini ", "on ne résiste pas à l'amour ", etc. L'ajout des adjectifs faery et unreflecting ne permet plus de considérer le lien de façon synthétique, ce qui rend N's $N$ encore moins probable. D'un point de vue générique, l'association paraît encore préconstruite : l'amour intellectualisé s'oppose depuis le début des sonnets à la magie de l'amour sensuel (on pense aussi à la dyade blakienne entre innocence et expérience). On note que unreflecting construit une sous-classe à partir de la notion <love>, l'adjectif (il n'existe pas de verbe correspondant to unreflect, et le sens n'est pas dérivé de love that [*un-]reflects) prend une valeur classifiante. L'épithète faery semble qualifier power en discours, en apportant un détail descriptif sur la notion. Cependant, il demeure possible de considérer que faery power constitue une sous-classe, celle du pouvoir mythique de l'innocence (perdue). Faery power prend une résonnance dans le genre lyrique et le romantisme, et pourrait même renvoyer au rôle de la muse. La connaissance de ces résonnances ferait de faery un adjectif classifiant, voire ce que Huddleston et Pullum (2012 : 448) nomment un composite nominal, où le premier terme (faery existe en tant que nom) garderait toutes ses dénotations notionnelles. deux prédicats centraux, de même sujet, organisés autour des verbes stand et think. Il leur est adjoint un circonstanciel de lieu : on the shore of the wide world, qui intègre un SP complément du nom shore, dénotant un lien à valeur d'inclusion. Aucun renvoi anaphorique (ni textuel, ni situationnel) vers l'amont du texte n'invite à tenir ce lien comme préconstruit. L'ensemble et ses constituants sont rhématiques, même si the wide world est fléché culturellement par son unicité référentielle et que l'ensemble entre encore une fois une résonance avec la tradition poétique : Nor the tide of pomp / That beats upon the high shore of this world (Shakespeare, Henry V, iv 1282 ).

Le verbe stand est un verbe positionnel rendu complexe par l'ajout de l'adjectif attribut alone à valeur dépictive. Le verbe think est employé intransitivement. Il prend le sens lexical fort de meditate. Il est suivi d'une proposition circonstancielle de temps, Till Love and fame to nothingness do sink, dans laquelle figure un dernier verbe occupant la place saillante de dernier mot du poème. Il s'opère ici une inversion stylistique qui permet de faire apparaître l'auxiliaire de support do non tonique. Frappés d'une majuscule dans certaines éditions du texte, Love et fame renvoient thématiquement à l'essence du sonnet lyrique et se font écho, ce qui facilite l'activation du topos romantique des réversibilités agentives : l'amour, thème lyrique par excellence, est un sentiment qui envahit, anime, autant qu'il se gagne et s'entretient, et la célébrité, motivation historiquement première des auteurs de sonnets, est poursuivie, provoquée, mais élit ses lauréats. Ces deux concepts se rejoignent ici négativement: ils sombrent dans les abysses, et avec eux, la muse inspiratrice. Par sa présence non contrainte, do pourrait 
pointer vers une mise en doute préalable. Cette valeur possiblement polémique n'est cependant activable que si l'on considère qu'elle se rattache aux attendus et aux promesses du genre tels qu'ils se présentent à un locuteur-poète. Le verbe intransitif sink [to nothingness] peut être suivi d'un circonstanciel indiquant la destination. Mais nothingness désigne un lieu métaphorique qui permet de configurer le SP comme un état résultant, la situation d'échec que redoute métatextuellement tout poète.

L'affaiblissement sémantique de l'agentivité du sujet est constant : behold, cease to be, my pen, with the magic hand of chance; les modaux épistémiques may et shall rendent compte de son manque de contrôle sur les choses; les multiples espaces et occurrences temporelles, qui densifient le monde extérieur, s'opposent à sa staticité et à sa solitude (I stand alone). En revanche, syntaxiquement, on assiste à un jeu entre promotion (dix occurrences $\mathrm{du}$ pronom sujet $I$, une $\mathrm{f}^{5}$ montée syntaxique) et déclassement (6 occurrences de I sur 7 sont précédées d'un subordonnant, when ou that, et l'on note aussi before my pen). La complexité syntaxique provient d'un enchâssement de subordonnées et de SP qui font que le sujet, s'il est textuellement saillant, reste pris dans l'espace de validation d'une subordonnée et dans le cadre spatial ou temporel de situations qui le dépassent. Cette complexité syntaxique offre un contraste saisissant avec la simplicité de I stand alone, qui combine promotion syntaxique du sujet (structure attributive simple sans modalité ni subordination) et affaiblissement agentif. Ce contraste est également utilisé à des fins génériques, puisqu'il marquera nettement la charnière avec la partie conventionnellement conclusive du sonnet. On note qu'un rapport quantitatif à visée de contraste s'insinue déjà dans la syntaxe.

\section{Répétitions et condensation}

\subsection{Répétition : la dialectique du même et du différent}

La répétition de termes, ici fréquente, tisse une dialectique, propre aux genres lyrique et romantique, du même et du différent.

Le subordonnant when apparaît trois fois. Sa répétition gagne un rôle structurel : il articule trois parties distinctes et permet à then qui lui répondra de mieux détacher la conclusion.

La préposition of apparaît à de nombreuses reprises, et nous avons vu qu'elle correspondait à des liens de natures diverses (possession, inclusion, attribution).

La répétition de termes s'accompagne souvent d'un changement de nature ou de fonction. Les propositions en that sont compléments de noms ou de verbes; la préposition upon introduit une première fois un attribut locatif (upon the night starr'd face localise symbols), puis le complément d'un verbe prépositionnel: look upon thee. Notons qu'ils suivent des verbes de sens proche : behold et look.

Le verbe have apparaît trois fois, mais have devant fears est lexical, has devant glean'd est auxiliaire. Dans have relish, c'est un élément d'une locution verbale. Son sens lexical est sélectionné par le nom déverbal relish, qui en fait un verbe de dynamisme hyperonymique, proche d'un verbe support.

31 Le terme high apparaît deux fois. Il est clairement adjectif dans high romance. Dans la première occurrence (high-piled books), il fonctionne avec pile. Il renseigne sur la manière dont sont empilés les livres. Une paraphrase serait : piled in such a way that they 
form a high pile. La structure décrit un état plus qu'elle ne renvoie à un procès avec agent implicite. Piled glisse du verbal à l'adjectival, et high, originellement adjectival ( $a$ high pile) prend un sens adverbial, testé par piled high above.

Un autre participe passé adjectival composé apparaît plus bas : the full-ripen'd grain. Il se construit à partir du verbe intransitif to ripen qui pose un argument affecté plutôt qu'agent. Toutefois, la main du cultivateur implicite demeure un agent facilitateur de ce mûrissement, ce qui permet un rapprochement entre la pile de livres / le stock de blé : leur fonction édifiante / nourricière pour la collectivité importe plus que l'individu à l'origine de leur empilement / mûrissement. High-piled et full-ripen'ed expriment tous deux l'accumulation, mais spatiale avec piled, temporelle avec ripen'd. La piste générique active le topos romantique que les auteurs, depuis l'antiquité, feraient œuvre commune, et que le patrimoine littéraire accumulé avec le temps s'érigerait spatialement telle une tour de Babel (high-piled). C'est dans sa conscience qu'il participe au patrimoine collectif que le poète puiserait alors un sentiment d'immortalité, à travers la survivance des livres, une postérité collective, sans ego. Tout comme sa plume (my pen), les livres gagneraient en agentivité : ils ne seraient pas empilés mais s'empileraient d'eux-mêmes. Ici encore, un topos générique (romantique : les œuvres, à commune fonction humanitaire, dépassent leurs auteurs) apporterait un élément de réponse à une interrogation syntaxique, en faisant pencher vers l'interprétation intransitive du verbe pile : books that have piled high plutôt que books that have been piled high.

Le terme fair pourait être rapproché de faery, d'autant que l'adjectif fair est suivi du nom creature, à la fois hyperonyme de human mais pouvant également désigner un être autre qu'humain, comme peut l'être une fée. Les deux adjectifs forment une paire minimale phonémique, mais ne possèdent pas le même étymon ni le même sens.

Le marqueur to est employé comme préposition introduisant par deux fois un complément caténatif : cease to be, live to trace. Mais le premier est au service d'une montée du sujet, l'autre non. Le dernier (to nothingness) est suivi d'un nom et renseigne sur un état résultant.

Le verbe think apparait à deux reprises. Il est d'abord employé comme verbe transitif direct et prend le sens de " croire ", puis de façon intransitive au sens de " réfléchir ».

Love apparaît d'abord comme nom commun, présenté dans une sous-classe notionnelle (unreflecting love) opposable à intellectualized love. Puis, dénué de déterminant et de modifieur, il renvoie à la notion en ce qu'elle s'oppose implicitement à fame. Le mouvement est inverse : une restriction référentielle, puis une référence absolue.

Le subordonnant temporel before est employé à deux reprises, sans différence notable. Toutefois, Before high-piled books, jette un temps un trouble sur sa valeur : suivi de highpiled books, de configuration spatiale, et du fait que le verbe hold soit reporté au vers suivant, le lecteur peut penser un temps que before est ici une préposition spatiale. On pourrait voir dans cette ambiguïté syntaxique momentanée un élément en faveur de la condensation entre temps et espace.

38 La répétition semble rompre le principe générique de quantité propre au sonnet (économie de moyens) et de ce fait, attire l'attention sur les termes répétés. Or, chacun est l'occasion d'une variation - la répétition elle-même servant à définir le thème qui permet de saisir les variations. En cela, Keats s'avère fidèle au genre lyrique dans sa version musicale. La boucle est bouclée: Keats s'écarte de certains principes 
morphosyntaxiques du genre, mais s'y réinscrit à travers une conformité d'ordre esthétique.

\subsection{Condensation de l'espace-temps} inversement. La haute pile de livres renvoie par exemple à la quête de postérité. À l'inverse, le grain mûri à terme remplit les greniers. La plume dessine des lignes horizontales de caractères (charact'ry) qui finiront en piles de livres érigées verticalement, mais elle court après le temps en tentant de le dominer. À l'inverse, la romance se spatialise en nuages et ombres, et les contingences prennent pour figure la main du hasard. La solitude du poète, debout à la lisière du monde, évoque sa solitude face aux deux principes essentiellement temporels que sont l'amour et la mortalité, et la douce créature aimée n'a de forme que le temps d'une heure. Quant au néant, dit par nothingness, il fait converger l'oubli et la disparition, un vide temporel, un autre spatial. Enfin, un poème n'est-il pas une tentative de spatialisation d'une expérience temporelle par le langage?

\subsection{Brouillage entre agentivité et passivité}

De la même façon que se brouillent les frontières entre temps et espace, celles entre agent et patient se compliquent. Les frontières habituelles entre "être ", "avoir » et « faire » semblent floues : la plume n'est plus l'instrument mais l'agent déclaré de l'acte poétique; les livres perdent leurs auteurs pour former un acquis collectif; le hasard subi, l'amour ressenti, la célébrité recherchée, prennent tous figure, existent de façon autonome; l'amour est un pouvoir qui nous submerge autant qu'il nous anime, il provient de l'autre autant que de projections personnelles, de même l'inspiration poétique.

41 Nous avons vu comment ces condensations prennent forme à l'échelle linguistique. On peut attribuer la condensation spatio-temporelle à l'impératif d'économie du sonnet, et le brouillage entre agentivité et passivité comme étant une problématique majeure du romantisme. La spécificité de l'auteur, si elle doit se mesurer par une comparaison au genre, pourrait provenir du fait que ces phénomènes sont particulièrement productifs dans le texte, et qu'ils lui donnent sa structure d'ensemble autant qu'ils se lisent à l'échelle des plus petits assemblages. Mais si la spécificité de ce texte devait provenir d'un écart vis-à-vis du genre, quel serait cet écart ?

\section{5. Étude de la quantité}

\subsection{Pluralité et quantité plurielle}

Le texte inclut cinq pluriels marqués morphologiquement : fears, high piled books, rich garners, huge cloudy symbols, shadows.

Trois de ces noms sont cognitivement configurables en tant que contenants, ils sont d'ailleurs chacun précédés d'un adjectif ou d'un syntagme adjectival. Ils expriment une pluralité seconde, accentuée par le modifieur : books précédé de high-piled présuppose un nombre suffisant de livres pour faire grande pile, mais aussi une pluralité interne de 
pages, de lignes, de mots, de caractères ; garners qualifié de rich présuppose que chaque grenier renferme une grande quantité de grains; cloudy symbols peut impliquer que chaque symbole possède différentes extensions, différentes interprétations, autant qu'un nuage compterait de protubérances.

Chacun de ces noms pluralisés possède par dénotation ou connotation les traits [+sombre] et [+utile] : l'obscurité du grenier, à fonction préservatrice ; l'encre noire des livres, offrant un nécessaire contraste au blanc de la page; l'opacité intrinsèque du symbole, génératrice de liberté interprétative ; le mystère impénétrable de la mort, qui suscite un obscur sentiment de peur exploitable comme source d'inspiration ; enfin l'ombre, empruntée métaphoriquement au nuage, qui offre nuance et contrepoint, et permet de saisir la lumière.

Chacun également renvoie à une singularité implicite: une peur est d'abord une obsession; un livre est une œuvre unique; un grenier est le bien précieux d'un agriculteur ; un symbole est une unité de signification, une ombre n'appartient qu'à soi. Chacun encore évoque indirectement l'isolement, voire la solitude, et ceci en contraste avec la pluralité décrite: la peur en tant qu'elle isole un être, la lecture en tant qu'activité solitaire, le grenier en tant que lieu usuellement désert; la signification en tant qu'interprétation personnelle; l'ombre en tant qu'elle renvoie à soi-même. Un premier paradoxe est que les pluriels forment un réseau qui renvoie à différents aspects de la singularité (obscurité, unicité, solitude).

D'autres pluralités implicites se glissent dans le texte : glean'd my teeming brain implique l'itération (glean'd) et l'abondance d'objet glanés (teeming); charact'ry est un nom collectif qui synthétise une pluralité de caractères. Encore une fois, ces termes évoquent la solitude (la cogitation propice à l'écriture), et une certaine obscurité liée au mystère de l'inspiration et du sens. Pourtant, un dernier, the night's starr'd face, évoque la lumière. Un pluriel, pléthorique, se révèle si l'on paraphrase le syntagme nominal : the night's face is filled/strewn with stars. En lieu et place des traits [+unique +sombre], la description penche plutôt ici vers l'infini et la lumière la plus intense qui soit. Une évocation de la solitude de l'homme reste possible: contempler les étoiles peut se concevoir comme un acte solitaire. Mais on penserait d'abord que la vision des étoiles est permise à chaque homme en tout point de l'univers, éternellement et simultanément. Ce contrepoint de lumière et d'union à l'autre offert par starr'd (pensons aux idiomes star-crossed lovers et wishing upon a star) offrirait alors une clé pour reconsidérer le corpus que forment les pluriels explicites : certes ils pouvaient évoquer la solitude, mais ils peuvent également se comprendre comme autant de traits d'union vers l'autre : partage d'expériences littéraires, de nourriture, de symboles communs, d'un même climat, de la même peur face à la mortalité.

Cloudy symbols, high piled books et rich garners dénotent une verticalité issue du quantitatif, à connotation (métonymique ou fonctionnelle) positive. Cette équivalence se manifestait déjà à l'échelle de l'adjectif rich, qui associe par métonymie le quantitatif au qualitatif. Ainsi, localisés en hauteur, les symboles et les livres deviennent précieux ; stocké en hauteur, le grain se trouve protégé du pourrissement. Configurables en tant que contenants, symbols, books et garners protègent ou renferment quelque chose de valeur (un sens, une histoire, le grain), qui peut nourrir, au sens propre ou figuré. Les grandes quantités amènent de grandes qualités (l'idée d'un patrimoine), a fortiori si les choses présentées en grande quantité contiennent elles-mêmes de grandes quantités, et/ou qu'elles occupent un espace vertical. Ces équivalences (multitude $=$ hauteur $=$ 
valeur) ne sont guère neuves, et semblent universelles, donc parfaitement romantiques (l'universel y prend valeur esthétique), ce que rappelle le syntagme nominal high romance. La spécificité du texte est de faire se répercuter ces équivalences structurales aux plus petites unités linguistiques.

$\mathrm{Ni}$ fears ni shadows n'évoquent la verticalité, synonyme de valeur positive. Par métaphore cognitive, la peur serait plutôt à associer à une stase ou à un recul, et les ombres, à une projection en deux dimensions d'une entité typiquement plane, d'extension horizontale. Fears [that I may cease to be] renvoie à la peur la plus tragique de l'être, et celle qui soulève le plus expressément les tourments d'un poète romantique; tandis que les ombres (never live to trace [the] shadows [of the huge cloudy symbols of a high romance]) renvoient finalement à la quintessence de la notion d'ombre, dont la propriété définitoire est peut-être l'intangibilité (exprimée par never live to trace). L'ombre est celle de high romance. Or, la notion romance est elle-même présentée dans sa quintessence, par high, mais aussi du fait que romance présuppose un dialogue entre l'auteur et le genre qu'il admire et dans lequel il cherche à inscrire son poème. Ainsi, fears et shadows, finalement, confirment l'équation qui associe grande quantité à grande qualité, mais indirectement: c'est surtout par connaissances génériques (le sujet étant dépeint comme étant un poète) qu'on peut les saisir comme désignant des notions de haute qualité, une qualité haute mais terrible puisqu'ils évoquent la mort et l'impuissance littéraire du sujet.

\subsection{Singuliers et quantité simple}

Grammaticalement, la détermination des noms au singulier recourt ici aux articles définis et indéfinis, aux déterminants possessifs et au génitif. Du point de vue de la référence, on peut ranger les occurrences selon trois continuums. Le premier concerne la nature des référents : non humain (shore), extension de l'humain (my pen), partie d'humain (my teeming brain), humain (I), humain idéalisé (fair creature, mention de faery). Le deuxième concerne la taille des référents: du microscopique (grain) au macroscopique (wide world), un continuum qui se jouait aussi dans la chaîne que forment charact'ry, symbols, books, pile. On peut penser au poème de Blake «To see a World in a Grain of Sand », lequel partage quatre noms avec le poème de Keats, et sans doute ce topos d'équivalence entre le micro- et le macroscopique :

To see a world in a grain of sand, (cf. vers 13 et 4$)$

And a heaven in a wild flower,

Hold infinity in the palm of your hand, (cf. vers 8)

And eternity in an hour. (cf. vers 9) singulier. Un autre point commun les unit, celui de la " haute qualité », exprimée par l'ajout d'un modifieur, d'essence adjectivale (high romance, magic hand, fair creature, wide world), adverbiale (full[y]-ripen'd grain), verbale (teeming brain) ou nominale (faery power); et dans Love et fame, par l'absence de tout dépendant. 
grammaire (the dans the night opère un fléchage culturel qui n'isole pas une nuit spécifique; when présuppose la récurrence), ou qu'elle provienne de connaissances génériques (dans les genres lyrique et romantique, la première personne devrait permettre une identification universelle). La possibilité de superposer les plans du micro et du macro est également rattachable à un genre (au sonnet, par ses impératifs de concision). Si typiquement on aurait tendance à associer l'emploi du singulier à une meilleure identification, on constate ici que chaque référent au singulier possède sémantiquement le trait de mystère et qu'il peut se dédoubler (il prend place dans la situation spécifique décrite mais peut figurer dans d'autres situations). De façon intéressante, ce paradoxe fait écho à celui qui frappe les pluriels, lesquels évoquaient la solitude en dépit de la multitude.

\subsection{Quantités basses à nulles}

53 Certains termes évoquent une quantité d'ordre temporel. Celle-ci sera toujours faible à nulle : of an hour, never (7 et 11), never [...] more.

D'autres évoquent une quantité qu'on pourrait dire existentielle, faible dans alone et shadows (quantité existentielle au sens où l'ombre ne représente que partiellement l'entité); nulle dans cease to be, nothingness, do sink. Dans ce dernier, on note que la quantité nulle d'existence impliquée par sink se voit renforcée par do.

\subsubsection{Quantité faible d'agentivité} validabilité moyenne. Cette valeur est à mettre au regard de la toute-puissance du hasard personnifié, et de l'inéluctabilité exprimée par shall. Avec may, l'énonciateur ne prend guère parti, en présentant la relation prédicative sous l'angle de l'équipossibilité. On pourrait parler d'un certain effacement assertif avec may. La valeur d'inéluctabilité de shall épistémique provient de ce qu'il encode une perte d'autonomie du sujet. Celuici est co-référent à l'énonciateur. Le modal shall marque un très haut degré de certitude quant à la validation de la relation prédicative mais aussi il implique que ce pronostic n'est pas de son fait : il s'impose à lui, il est dicté par l'ordre des choses.

May et shall présentent par des opérations opposées (sous-assertion, sur-assertion) un locuteur ne contrôlant pas les événements, tour à tour peu certain des choses, non responsable de la prévision, ou tributaire du hasard souverain. Un jeu de quantification 
apparaît par le mouvement de may à shall : degrés d'assertion différents, et pourtant même effet final : mettre en contraste une forte présence énonciative et une absence de contrôle sur les choses.

\section{Conclusion}

60 Ce poème est, pourrait-on dire, un poème du haut degré, comme le laisse à penser la répétition de high à seulement trois vers d'écart (3 et 6). Les quantités plurielles, singulières, mais aussi les quantités faibles et nulles, se voient toutes associées - de façons diverses - à la haute qualité (de noblesse ou de mystère, d'espoir ou de désespoir, de plein ou de vide). En appliquant à chaque endroit une équivalence entre quantité et qualité, entre spécifique et générique, micro et macro; en exploitant l'économie de la métonymie et en recourant aux métaphores universelles, du style UP is good, IN is safe, valuable (Lakoff, 2003), le poète accommode avec succès son propos à la forme contraignante du sonnet.

61 Ce poème, tardif dans l'histoire du sonnet mais aussi du romantisme, possède bien sûr des traces d'intertextualité (pen et hand se retrouvent dans Sidney, Sonnet 70 ; creature et Fate dans Donne, $5^{\text {ème }}$ sonnet dans « La Corona ", shore et fame dans Daniel xxxi, etc.). Plus intéressant est le fait que ce texte convoque des thèmes tour à tour propres au lyrisme, au sonnet et au romantisme. Encore plus pertinent est le fait qu'elles apparaissent sous la forme de topoï, c'est-à-dire sous la forme d'une dialectique ou d'un schéma argumentatif traditionnels, indice de leur généricité. Mais ici, l'originalité de Keats et sa spécificité stylistique tient à ce que cette dialectisation, qui frappe des thématiques issues de multiples genres, passe systématiquement par un rapport d'équivalence entre le quantitatif et le qualitatif, dont le rôle structurant est si poussé qu'il concerne également la composition syntaxique du poème.

L'auteur décline le principe de quantité aux plans syntaxique, référentiel, agentif, énonciatif, et au final, propose une dernière équivalence, plus personnelle: toute quantité, nulle à forte, est associée à une forme d'absence, laquelle est promue en qualité haute. La grande quantité est associée à la solitude (plus qu'au foisonnement), et la quantité simple au mystère (plus qu'à l'identifiable). Le poème entier tente d'appréhender l'absence, et de la transformer en objet esthétique. Puisque le langage est une mise en présence verbale d'un référent absent, puisque les livres dépossédés de leurs auteurs forment patrimoine, c'est en créant sa poésie autour d'un constat d'absence que Keats tirera son œuvre du néant.

La répétition, tout comme l'abondance paradoxale des termes de quantités faibles à nulles pose un défi aux limites spatiales de la forme, mais au final, ce conflit entre le temps de sa subjectivité et l'espace générique constitue le propos du texte. Ce poème est original à deux titres : en ce qu'il cristallise de façon incroyablement condensée les attendus provenant tout à la fois du lyrisme, du sonnet, et du romantisme, et en ce qu'il parvient à faire de la quantité un vecteur qui alimente à la fois sa thématique d'ensemble, ses constructions linguistiques discrètes, ses problématiques, et une conscience de la forme employée. Le paradoxe de la faible quantité amenant une grande qualité révèle en creux la conscience générique de Keats: plus le sujet est humble, et plus le lyrisme s'accroît, plus il se désagentive (par ses peurs et son impuissance déclarée), et plus il devient un héros romantique, plus l'absence et le néant sont exposés, et plus la valeur évocatrice du langage s'affirme. 
64 l'énoncé et des éléments thématiques et morphosyntaxiques attribuables à des genres
ici en imbrication a permis de dégager la spécificité stylistique de l'auteur en ce qu'elle
se distingue de traditions de tous horizons : les "traces " que laissent ce dialogue
conscient entre Keats et les genres sont au cœur de l'esthétique de ce texte. Une valeur
esthétique qui a fait que, bien que construit autour d'un aveu d'impuissance littéraire,
ce sonnet est devenu aujourd'hui un texte-événement non pas d'un genre, mais de la
littérature britannique.

\section{BIBLIOGRAPHIE}

ANSCOMBRE, J.-C., éd. (1995), Théorie des topoï, Paris, Editions Kimé.

BAKHTINE, M., 1984 [1952-1953], « Les genres du discours [language] », Esthétique de la création verbale, Paris, Gallimard, p. 263-308.

BARTLETT, J., 2016 (1894), A Complete Concordance to Shakespeare, London, The Macmillan Press Ltd.

BOUSCAREN J. et CHUQUET J., 1987, Grammaire et textes anglais : guide pour l'analyse linguistique, Paris, Ophrys.

CURRAN, J., 1986, Poetic Form and British Romanticism, OUP, New York.

DUFF, D., 2009, Romanticism and the Uses of Genre, Oxford, OUP.

FONAGY, I., 1966, « La langue poétique : forme et fonction », Problèmes du Langage, Paris, NRF/ Gallimard, p. 72-113.

GIRARD-GILLET, G., 2016, « Aspectual Verbs : a study of cease and continue », Anglophonia 22, http://journals.openedition.org/anglophonia/1036.

GITTINGS, R., 1966, Selected Poems \& Letters of Keats, Oxford, Heinemann Educational Books Ltd.

GRILLO, S. V. C, 2007, «Epistémologie et genres du discours dans le cercle de Bakhtine », Linx, Nanterre, n. 56, p. 19-36.

HUDDLESTON, R. \& PULLUM, G. K., 2012 (2002), The Cambridge Grammar of the English Language, Cambridge, Cambridge UP.

KOCH, P., 1997, « Diskurstraditionen : zu ihrem sprachtheoretischen Status und ihrer Dynamik », in B. Frank, T. Haye, D. Tophinke (eds.), Gattungen mittelalterlicher Schriftlichkeit, Tübingen, Narr, pp. 43-79.

LAKOFF, G., JOHNSEN, M., 2003 (1980), Metaphors we Live By, London, the Chicago UP.

LAPAIRE, J.-R. \& ROTGE, W., 1991, Linguistique et grammaire de l'anglais, Toulouse, PU du Mirail.

PERLMUTTER, D., 1970, « The Two Verbs Begin », in J. Roderick \& P. Rosenbaum (eds), Readings in English Transformational Grammar, Waltham, Blaisdell, 107-119.

Linx, $78 \mid 2019$ 
RASTIER, F. \& PINCEMIN, B., 1999, « Des Genres à l'intertexte », Cahiers de praxématique, 23, p. 90-111.

ROSS, J. R., 1969, « Auxiliaries as Main Verbs », Studies in Philosophical Linguistics, Series One, Carbondale, Great Expectations Press.

SPILLER, M. R. G., 1992, The Development of the Sonnet, An Introduction, London, Routledge.

WURMBRAND, S., 1999, « Modal verbs must be raising verbs », West Coast Conference on Formal Linguistics (WCCFL) 18, Somerville, MA, Cascadilla Press, p. 599-612.

\section{NOTES}

1. On note à ce titre qu'en tant que romantique tardif, Keats possédait sans nul doute une forte conscientisation des normes mais aussi de l'historicité des genres qu'il souhaitait styliser. Lecteur admiratif des grands poètes, Keats usa d'ailleurs d'une variété de formes compositionnelles finalisées (ode, sonnet, poème narratif, etc.).

2. Interrogé personnellement sur la question le 4 décembre 2015, Jean-Jacques Lecercle propose d'exclure des propriétés d'un genre les thèmes de nature idéologique, qui sont à rattacher à la doxa - leur transversalité, ou trans-généricité, pouvant constituer un indice du statut idéologique.

3. Nous reproduisons ici la version éditée par Gittings (1966 : 43).

4. Si l'on interprète l'énoncé comme une réduction de live long enough to trace, to introduirait alors l'élément repère de la comparaison figurant dans une la subordonnée comparative d'équivalence, ce qui n'est toujours pas une infinitive de but.

5. Une seule avec cease, mais si l'on accepte de considérer les auxiliaires modaux comme relevant de la catégorie des verbes, ainsi que le préconise Ross (1969), dans ce cas, dans la mesure où les épistémiques may et shall n'assignent pas de rôle-thêta au sujet de surface, il serait envisageable comme le fait Wurmbrand (1999) de les considérer eux aussi comme des verbes à montée.

\section{RÉSUMÉS}

Le sonnet lyrique «When I have fears » de John Keats met en scène un poète intra-diégétique tourmenté par la perspective d'une faillite littéraire. Entièrement retourné sur lui-même, ce poème semble construire sa signification autour d'un dialogue avec les genres. Mais qu'est-ce qu'un genre? Dans un premier temps, cet article tentera de définir la notion, afin de tester les entrées que sont le sonnet, le lyrisme et le romantisme. La conception bakhtinienne du genre constituera le point de départ de notre étude, laquelle propose de partir à la recherche des traces que laisse le dialogue entre l'énoncé et le genre dans lequel il s'inscrit nécessairement. Celles-ci révéleront de la part de l'auteur une forte conscientisation des principes relatifs à ces entrées, faisant dire qu'ils sont, au moins pour l'auteur, à voir comme des genres. Nous partirons de la syntaxe, et verrons comment les genres informent celle-ci, tant dans l'encodage que dans le décodage. L'étude détaillée de la syntaxe montrera comment l'énoncé se plie aux principes de la forme du sonnet (notamment par la condensation) mais aussi comment il les utilise pour marquer certaines spécificités (notamment par la répétition). Nous verrons comment s'opèrent 
déjà par la syntaxe de subtiles activations de topoï génériques, et comment le principe de quantité est déjà exploité de façon signifiante. La deuxième partie de l'article sera entièrement consacrée à l'étude de la quantité, que nous déclinerons selon plusieurs domaines. Nous rapprocherons quantité et qualité, et verrons comment le texte tire sa spécificité d'une mise en équivalence systématique et multimodale entre quantité et qualité, un motif structurant personnel que l'auteur applique au final à tous les principes thématiques et morphosyntaxiques des genres qu'il emprunte.

The lyrical sonnet « When I have fears » by John Keats stages an intra-diegetic poet tormented by the prospect of his literary failure. Entirely turned on itself, the meaning of this poem seems to be constructed on the basis of a dialogue with genres. But what is a genre? The first aim of this paper is to try to define what a genre is, with a view to assessing to what extent the sonnet, the lyric, and romanticism lend themselves to a configuration as genres. In line with Bakhtin's conception of genres, we will look for the traces of a dialogue between the utterance and the genre that it necessarily belongs to. These will reveal in Keats a hyperconsciousness toward the generic principles pertaining to the sonnet, the lyric, and romanticism. To him at least, they are to be seen as genres. We will then see how genres inform syntax, both in the encoding and the decoding processes. The text complies with the formal requisites of the sonnet (notably by condensation), but it also departs from them to gain its specificity (notably by repetition). We will see how some syntactic options facilitate the activation of certain generic topoï, and how the principle of quantity already plays a significant role in the syntax of the text. The second part of our study will be devoted to quantity, which will be broken down into different planes. By studying quantity in conjunction with quality, we will see how the specificity of the text may come from the way it draws a systematic and multimodal relation of equivalence between quantity and quality, so that this equivalence comes to act as an original structural motif that the author applies in fact to all the thematic and morphosyntactic principles of the genres he borrows.

\section{INDEX}

Mots-clés : Romantisme, sonnet, lyrisme, John Keats

Keywords : Romanticism, sonnet, Lyricism, John Keats

\section{AUTEUR}

\section{STÉPHANE KOSTANTZER}

Université de Strasbourg, EA1339 LILPA, Fonctionnements Discursif et Traduction 